\title{
Reconstruction of the roadway coverage parameters from radar probing measurements
}

\author{
A. Krainyukov \\ Faculty of Computer Science and Electronics, Transport and \\ Telecommunication Institute, Latvia
}

\begin{abstract}
This work has focused on the development of an approach to the solution of the structural inverse problem of radar subsurface probing of roadway coverage. To solve the inverse problem in the frequency domain by the method of comparison, iterative procedure is used for the aim of function minimization. The direct problem of radar subsurface probing is based on the linear system response functions for the radar antenna system and on the equations for wave propagation in horizontally multilayered roadway coverage. The inverse problem has been investigated numerically. A genetic algorithm is used to investigate the global minimum of the aim function. Statistical assessment of the reconstruction accuracy under various conditions has been done. The aim function of the inverse problem of radar probing of roadway coverage was investigated.
\end{abstract}

Keywords: radar probing measurements, reconstruction of the roadway coverage, inverse problem of radar probing, genetic algorithm.

\section{Introduction}

Radar subsurface probing is widely used for performing scientific and practical research. It allows one to find the hidden objects. Roadway coverage is a complex multi-layered construction. It is well known that in different seasons of the year and in different environmental conditions the preservation of road cover depends not only on usage but also on different climate-related factors. As a result different processes take place within roadway coverage: the appearance of voids, changes in dampness of the soil, changes in the ability of the soil to filter water, etc. Timely identification of these processes allows one to make a timely 
decision about the necessary actions for optimization of exploitation, upkeep and reconstruction of the road cover [1].

Nowadays to research the inner structure of roadways subsurface radar probing methods are widely used $[2,3]$. In spite of substantial achievements in research and development of the radar probing methods for roadway coverage, there are some problems to improve the interpretation of radar probing data. The efficiency of the radar probing heavily depends on the algorithms and the methods of signal processing, reflected from the roadway coverage. Traditional interpretation methods of the roadways subsurface radar probing results do not provide the required precision and effectiveness of roadway monitoring. Subsurface radar profiles provide only a qualitative picture of the roadway condition. Only an experienced specialist can restore the approximate structure of the roadway coverage under investigation.

This is not enough for radar monitoring of roadway coverage, since the radar probing of roadways has specific features. Ensuring high reliability and efficiency of roadway coverage radar monitoring is a challenge. It is necessary to perform reconstruction of electro-physical parameters of roadway coverage with detection and identification of inner zones and objects.

\section{Inverse problem of roadway coverage radar probing}

Reconstruction of the geometrical and electrical parameters can be performed by using the secondary processing of radar probing signals, identification and object recognition. These problems can be solved by using various computational methods and algorithms. However, the applied computational methods and algorithms have to provide the required accuracy of the results of radar probing interpretation of the roadway coverage and the diagnosis of the internal structural elements condition.

Reconstruction of the geometrical and electro-physical characteristics of the object under exploration based on the radar measurements results is an inverse problem of radar probing, which is, in general, ill-posed and not unique. To solve this problem, different methods of comparing the direct solution of an assumed subsurface structure with the measured data of radar probing are usually applied. The solution of the inverse problem by the comparison method can be found both in the time and the frequency domain.

An inverse problem for the radar probing of roadway coverage is usually a structural inverse problem. Prior information is used in order to solve structural inverse problem. Prior information can be obtained from the appropriate road documentation. Prior information allows us to define the roadway coverage structure and to create its electrical model, which is then used for solving the direct problem of the radar probing.

In this work the inverse problem of roadway coverage radar probing is solved in the frequency domain using the vector of parameters $\vec{P}=\left\{p_{1}, p_{2}, \ldots, p_{n}\right\}$, where $\mathrm{n}$ - the number of model layers and $p_{i}$ - electro-physical parameters of the model i - layer. Electro-physical parameters of each layer are: thickness $h$, 
conductivity $\sigma$ and relative dielectric constant $\mathcal{E}$, and the range of possible values of these parameters [4].

When solving the inverse problem of subsurface probing it is very important to rationally select the type of informative characteristics, the quantity and the area of the original data in order to calculate the aim function. While solving the inverse problem of radar probing in the frequency domain by the method of comparison, the aim function $\Phi$ looks the following way:

$$
\Phi=\frac{1}{n_{\max }} \sum_{i=0}^{n_{\max }}\left|B_{e}\left(\omega_{i}, \vec{P}\right)-B_{t}\left(\omega_{i}, \vec{P}_{M}\right)\right|^{2}
$$

where $\mathrm{n}_{\max }$ is index of the spectral component with frequency $\mathrm{f}_{\max } ; B_{e}\left(\omega_{i}, \vec{P}\right)$ is value of informative characteristics, it is derived from the signal reflected by roadway coverage, which is characterized by vector $\vec{P} ; B_{t}\left(\omega_{i}, \vec{P}_{M}\right)$ is calculated theoretical value of the informative characteristics for solving the direct problem of subsurface radar probing. To calculate $B_{t}\left(\omega_{i}, \vec{P}_{M}\right)$ the vector of parameters $\vec{P}_{M}$ is chosen, it belongs to the set of allowed values of parameters $\vec{P}_{P O S}$. The solution of the inverse problem is the vector of parameters $\vec{P}_{M}$. In order to find vector $P_{M}$, one has to find the global minimum of the aim function $\Phi$.

Modular or complex values of the reflected signal's spectral density or coefficient of medium reflection can be used as an informative characteristic. In this work the reflected signal's complex spectral density $\dot{S}_{e}\left(\omega_{i}, \vec{P}\right)$ and modular spectral density $\left|\dot{S}_{e}\left(\omega_{i}, \vec{P}\right)\right|$ are used as informative characteristic $B_{e}$.

The aim function $\Phi$ depends on informative characteristic and a great number of its arguments in a complicated way. Along with a global minimum aim function $\Phi$ has a great number of local minimums. Inverse problem has been investigated numerically by using electromagnetic model of roadway coverage and direct problem model of subsurface radar probing. Iterative procedure was chosen for the search of global minimum of aim function $\Phi$ by using genetic algorithm [4].

\section{Models and conditions for inverse problem solving}

\subsection{Direct problem model of subsurface radar probing}

A typical subsurface radar system has three main components: a transmitter and a receiver are directly connected to their antennas, and a control unit with a display [3]. The transmitting antenna radiates a short high-frequency 
electromagnetic (EM) pulse into the inspected medium, where it is refracted, diffracted and reflected primarily as it encounters changes in dielectric permittivity and electric conductivity. Waves that are reflected back by the probed medium induce signals in the receiving antenna, and are recorded as digitized signals for display and further analysis.

According to that, the signal forming channel for subsurface radar probing in frequency domain may be presented as it is shown in fig. 1 [4].

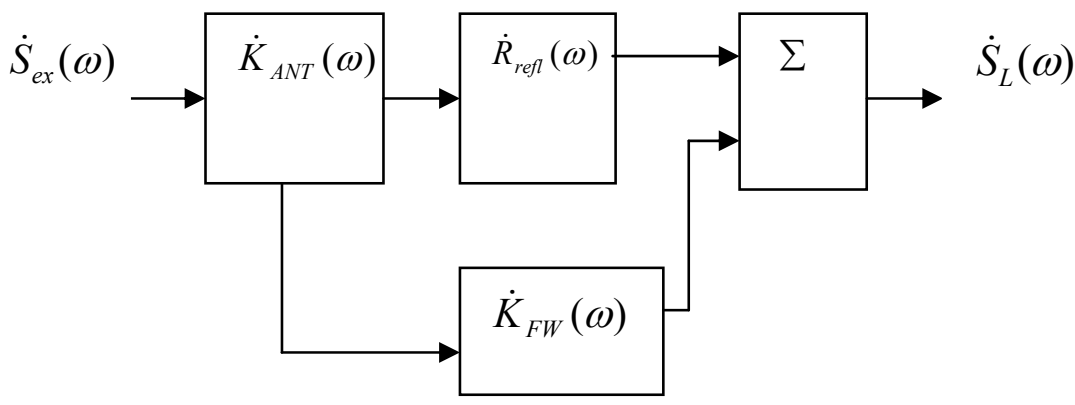

Figure 1: $\quad$ Signal forming channel for subsurface radar probing in frequency domain.

There $\dot{K}_{A N T}(\omega)$ is a complex transfer function of the antenna system, $\dot{R}_{\text {refl }}(\omega)$ is a reflection coefficient of the object under inspection, and $\dot{K}_{F W}(\omega)$ is a complex transfer function of the antennas directly coupled in the EM field; $\dot{S}_{e x}(\omega)$ is the spectrum of the signal, which is used for impact excitation of the transmitting antenna, $\dot{U}_{L}(\omega)$ is the spectrum of the signal across the load resistance of the receiving antenna.

As a result, complex transfer function of radar subsurface probing model may be represented in the following way:

$$
\dot{K}_{R A D}(\omega)=\dot{S}_{L}(\omega) / \dot{S}_{e x}(\omega)=\dot{K}_{F W}(\omega)+\dot{K}_{A N T}(\omega) \dot{R}_{r e f l}(\omega)
$$

It depends on the conditions of the subsurface radar probing, as well as on the geometry of the antennas location. To get the expression $\dot{K}_{R A D}(\omega)$ the following features of the subsurface radar probing were taken into account:

- both radar antennas are linear vibrators;

- the radar antennas are located in the air and are parallel to each other;

- the height of antennas location $\mathrm{H}$ over upper medium boundary is sufficiently small $(H \approx 0)$. 
Then complex transfer function of the antenna system $\dot{K}_{A N T}(\omega)$ may be presented as:

$$
\begin{aligned}
\dot{K}_{A N T}(\omega)= & -j \frac{30 k_{0} \dot{L}_{e f f}^{2}(\omega) R_{L}}{\sqrt{d^{2}+\left(2 h_{2}\right)^{2}}\left[R_{L}+\dot{Z}_{\text {in }}(\omega)\right]^{2}} \\
& \cdot \frac{\sqrt{\dot{\varepsilon}_{2}(\omega)} \cos \theta_{0}}{\sqrt{\dot{\varepsilon}_{2}(\omega) \sin ^{2} \theta_{0}}-\sqrt{\dot{\varepsilon}_{2}(\omega)} \cos \theta_{0}}
\end{aligned}
$$

where: $j=\sqrt{-1}$ is image unity; $l$ is half length of linear antennas; $d$ is the distance between the radar antennas; $k_{0}=\omega / c$ is the wave number for free space; $\omega=2 \pi f$ is the angular frequency of monochromatic wave with liner frequency $f ; \theta_{0}=\operatorname{arctg}\left(d / 2 h_{2}\right)$ is the incident angle; $h_{2}$ and $\dot{\varepsilon}_{2}$ are thickness and complex permittivity of the inspected layer; $\dot{Z}_{i n}(\omega)$ is input impedance of radar antennas; $R_{L}$ is load resistor of the receiving antenna; $\dot{L}_{\text {eff }}(\omega)$ is effective antennas length. $\dot{L}_{e f f}(\omega)$ is expressed as

$$
\dot{L}_{e f f}(\omega)=\frac{2}{\dot{k}_{L}} \cdot \frac{1-\cos \dot{k}_{L} l}{\sin \dot{k}_{L} l},
$$

where: $l$ is half length of linear antennas; $\dot{k}_{L}$ is the complex wave's number[5], which depends on antennas diameter a, antennas high $\mathrm{H}$ over upper boundary of inspected medium, and complex refraction coefficient $\dot{n}=\sqrt{\dot{\varepsilon}_{2}(\omega)}$.

In accordance with [4], complex transfer function of the antennas directly coupled field is expressed as

$$
\begin{aligned}
\dot{K}_{F W}(\omega) & =j \frac{30 k_{0} \dot{L}_{e f f}^{2}(\omega) R_{L}}{\left(\sqrt{\dot{\varepsilon}_{2}(\omega)}-1\right) d\left[R_{L}+\dot{Z}_{i n}(\omega)\right]^{2}} \\
& \cdot\left[\sqrt{\dot{\varepsilon}_{2}(\omega)} e^{-j k_{0} \sqrt{\dot{\varepsilon}_{2}(\omega)} d}-e^{-j k_{0} d}\right] .
\end{aligned}
$$

Reflection coefficient $\dot{R}_{\text {refl }}(\omega)$ is expressed as a complex reflection coefficient for oblique incidence of plane wave on the upper boundary of the inspected medium. Equations (2), (3) and (5) have been used for further direct problem solution of roadway coverage radar subsurface probing in the frequency domain in order to calculate $\dot{S}_{L}(\omega)$. 


\subsection{Electromagnetic properties modelling of roadway coverage}

The electro-physical parameters of the roadway coverage layers were modeled taking into account that the roadway coverage layers are composed of such materials as asphalt, concrete, crushed stone, crushed slag, sand and others. The number of the layers can vary but the electro-physical characteristics of some layers can be very similar or even equal. Roadway coverage layers are placed between two semi-infinite spaces where the upper semi-infinite space is air and the lower one is subgrade. Two models of the roadway were used in carrying out this research: double-layer and three-layer models. Electro-physical parameters of partial double-layer model medium are presented in table 1 .

Table 1: Electro-physical parameters of partial model medium.

\begin{tabular}{|c|c|c|c|}
\hline \multirow{2}{*}{$\begin{array}{c}\text { Partial medium of } \\
\text { model }\end{array}$} & \multicolumn{3}{|c|}{$\begin{array}{c}\text { Electro-physical parameters of partial } \\
\text { model medium }\end{array}$} \\
\cline { 2 - 4 } & $\varepsilon^{\prime}$ & $\sigma, \mathrm{s} / \mathrm{m}$ & $\mathrm{h}, \mathrm{m}$ \\
\hline Air & 1.0 & 0 & $\infty$ \\
\hline First layer & 2.6 & 0.00015 & 0.3 \\
\hline Second layer & 2.6 & 0.001 & 0.4 \\
\hline $\begin{array}{c}\text { Lower semi-infinite } \\
\text { space (subgrade) }\end{array}$ & 15.0 & 0.05 & $\infty$ \\
\hline
\end{tabular}

\subsection{The characteristic features of solving the inverse problem by using the genetic algorithm}

Searching for the global minimum by means of the genetic algorithm takes a lot of time and may even turn out to be impossible. It has been suggested, that genetic algorithm is used to study such value of the aim function $\Phi$, which is not more the value of threshold $\alpha$. Therefore, value of threshold $\alpha$ has a substantial influence on the inverse problem solution while using genetic algorithm. Use of great value of threshold $\alpha$ does not allow one to reconstruct the electro-physical parameters of roadway coverage with enough accuracy. Diminishing threshold $\alpha$ increases the time of inverse problem decision substantially.

The aim function $\Phi$ is a power parameter, because value of threshold $\alpha$ may be calculated by using mean power of spectral constituents of $\dot{S}_{e}\left(\omega_{i}, \vec{P}\right)$, used for the calculation of aim function $\Phi$. If the number of spectral components is $n_{\max }$ the average mean power of those spectral components $\dot{S}_{e}\left(\omega_{i}, \vec{P}\right)$ is expected to be as follows:

$$
P_{a v}=\frac{1}{n_{\max }} \sum_{i=0}^{n_{\max }}\left|\dot{S}_{e}\left(\omega_{i}, \vec{P}\right)\right|^{2},
$$


but the value of $\alpha$ is set as

$$
\alpha=\frac{P_{a v}}{K},
$$

where $K$ is a dimensionless coefficient, set by the user. Nevertheless, the value of $K$ must be chosen so that the specified reconstruction accuracy of roadway coverage parameters is taken into account.

Values of the electro-physical parameters of the layers of the road coverage can vary in the limits of up to 1.5 times. Therefore for the formation of chromosome population of genetic algorithm the possible values range of every electro-physical parameter is from $0,75 \mathrm{v}$ to $1,25 \mathrm{v}$, where $\mathrm{v}$ is the value of each specific parameter (see table 1). The range of search for each parameter was $50 \%$ of its model value.

\section{Results of roadway coverage parameters reconstruction}

For the investigation of the inverse problem algorithm that had been considered above, software application has been developed, which is able:

- to model the structure and the electro-physical parameters of the road coverage;

- to find the solution of the direct problem of the radar probing;

- to find the solution of the inverse problem of the radar probing by using the genetic algorithm;

- to investigate the aim functions, etc.

All the results mentioned below have been obtained after applying this software.

The model signal on the output of the receiving antenna, which was obtained with the help of equations (2)-(5) and parameters, is presented in table 1.

The probing signal was generated by the impact excitation of the transmitting antenna for $\mathrm{H}=0,01 \mathrm{~m}, \mathrm{l}=0,5 \mathrm{~m}$ and $\mathrm{d}=0,5 \mathrm{~m}$ and was a decaying harmonic oscillation with the central frequency $100 \mathrm{MHz}$. Impact excitation pulse of the transmitting antenna was triangular pulse, duration of which was equal to $2 \mathrm{~ns}$. Almost the whole energy (99.9\%) of the signal, reflected from the model medium, is located within the band $10-500 \mathrm{MHz}$.

The solution of the inverse problem is vector $\vec{P}_{M}$, it was further used to find the relative error of reconstruction for each of roadway coverage parameters. In order to define the optimal conditions for the generic algorithm to be used to solve the inverse problem of radar subsurface probing we tried to find out how these values were influenced by the following factors:

- the coefficient $\mathrm{K}$, defining the threshold of the acceptable solution $\alpha$;

- frequency range of the reflected signal spectrum used, limited by its maximal frequency $f_{\max }$.

To obtain statistical assessment solutions of the inverse problem about 100 examples were used. The value of $K$ in our calculations was changed within the range of 100 to 5000 with fixed $f_{\max }=500 \mathrm{MHz}$. 
Figures 2 and 3 show influence coefficient $\mathrm{K}$ on the relative error (upper figure) and the relative root-mean-square (RMS) error (lower figure) of reconstruction of electro-physical parameters of the first layer.
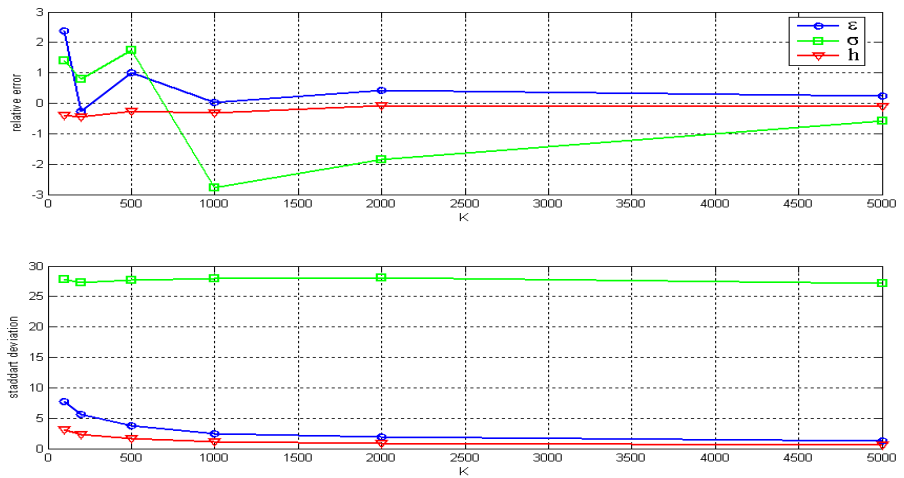

Figure 2: $\quad$ Influence of value $K$ on accuracy reconstruction of the first layer parameters by using $\dot{S}_{e}\left(\omega_{i}, \vec{P}\right)$.
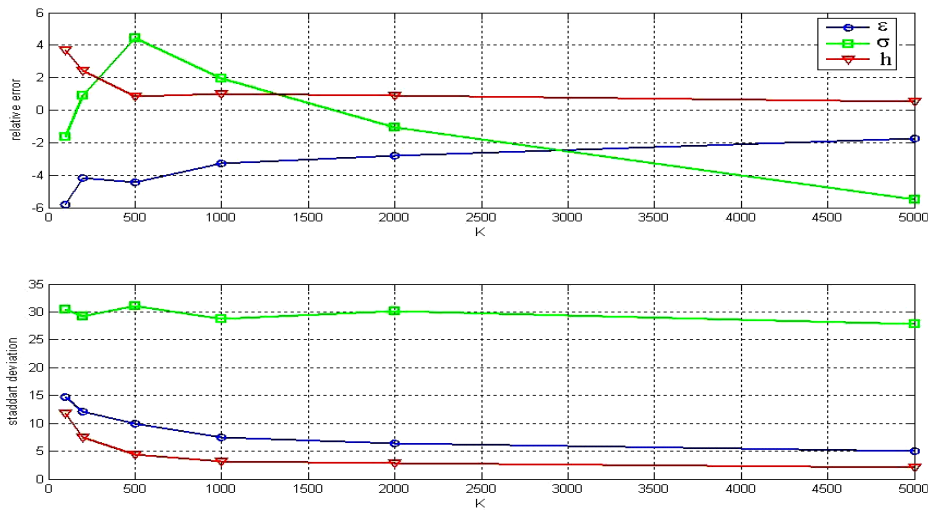

Figure 3: Influence of value $K$ on accuracy reconstruction of the first layer parameters by using $\left|\dot{S}_{e}\left(\omega_{i}, \vec{P}\right)\right|$.

When using $\dot{S}_{e}\left(\omega_{i}, \vec{P}\right)$ the relative errors of reconstruction $\varepsilon_{1}^{\prime}$ and $h_{1}$ are less than when using $\left|\dot{S}_{e}\left(\omega_{i}, \vec{P}\right)\right|$, and are, in fact, close to 0 . When $K \geq 1000$ the relative errors of reconstruction $\varepsilon_{1}$ and $h_{1}$ slightly decrease.

Dependencies of relative RMS error for the first layer parameters show that the increase of $\mathrm{K}$ will result in a smaller range of possible values of $\varepsilon_{1}^{\prime}$ and $h_{1}$. The range of possible values of $\sigma_{1}$ remains unchanged with the increase of $K$; therefore the values of relative RMS error for $\sigma_{1}$ are stable and significant. For this reason dependencies of $\sigma_{1}$ have a chaotic character. 
Influence of value $\mathrm{K}$ on relative RMS errors of the second layer parameters reconstruction is similar to those of the first layer. The difference is in higher values of relative RMS errors for $\varepsilon_{2}^{\prime}$ and $h_{2}$. Correspondingly the relative error for $\varepsilon_{2}^{\prime}$ and $h_{2}$ is higher, as it is shown on figures 4 and 5 , although they decrease with the increase of $\mathrm{K}$ when using $\left|\dot{S}_{e}\left(\omega_{i}, \vec{P}\right)\right|$ (fig. 5). When using $\dot{S}_{e}\left(\omega_{i}, \vec{P}\right)$ the relative errors for $\varepsilon_{2}^{\prime}$ and $h_{2}$ are less than $1 \%$ already when $K=1000$ (fig. 4). Dependence of $\sigma_{2}$ has also chaotic character as RMS error for $\sigma_{2}$ is independent of $K$.

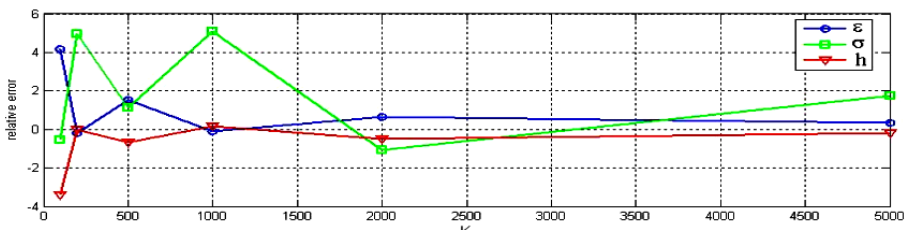

Figure 4: $\quad$ Influence of value $K$ on accuracy reconstruction of secondary layer parameters by using $\dot{S}_{e}\left(\omega_{i}, \vec{P}\right)$.

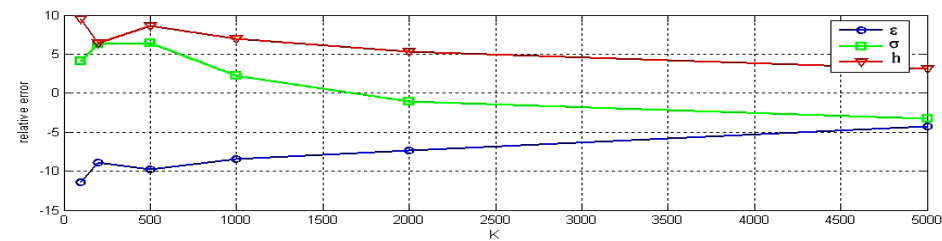

Figure 5: Influence of value $K$ on accuracy reconstruction of second layer parameters by using $\left|\dot{S}_{e}\left(\omega_{i}, \vec{P}\right)\right|$.

Influence of value $K$ on accuracy reconstruction of lower semi-infinite space (subgrade) parameters is shown on figures 6 and 7. Increase of value $K$ leads to decrease of RMS errors for $\varepsilon_{3}^{\prime}$ and $\sigma_{3}$ and therefore to decrease of relative errors of reconstruction. When $K$ is more than 1000 the relative errors hardly change. Values of $\varepsilon_{3}^{\prime}$ and $h_{3}$ are reconstructed with relative error less than $1 \%$ already when $K=1000$.

The dependence of the aim function on electro-physical parameters of the roadway coverage has been investigated. In order to demonstrate the dependence of the aim function on electro-physical parameters in a three-dimensional form two partial electro-physical parameters have been selected for calculations of the aim function. To calculate the value of the theoretical spectral density $\dot{S}_{t}\left(\omega_{i}, \vec{P}_{M}\right)$ the values of two of the electro-physical parameters of one of the roadway coverage layers have been changed, while keeping the remaining 6 constant and equal to the model ones. The range of changes of the chosen 
parameters has been symmetrical to the model values and equal to them. Dependencies of aim function $\Phi$ on dielectric permittivity $\varepsilon_{1}$ of the first layers and their thickness $h_{l}$ are shown on figures 8 and 9 .

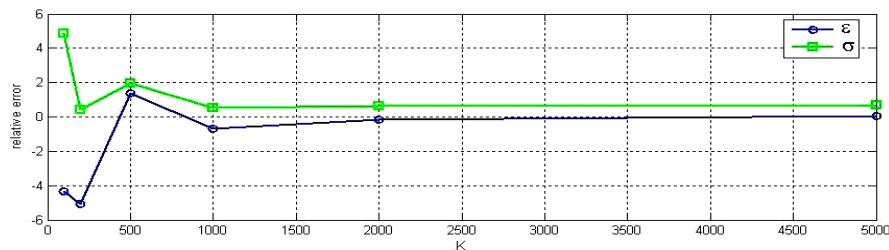

Figure 6: Influence of value $K$ on accuracy reconstruction of lower subgrade parameters by using $\dot{S}_{e}\left(\omega_{i}, \vec{P}\right)$.

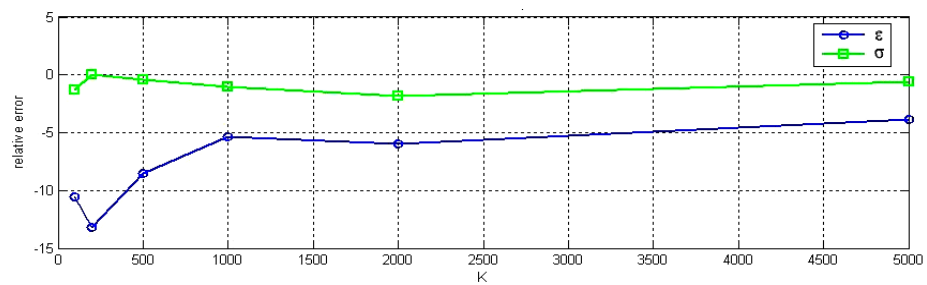

Figure 7: Influence of value $K$ on accuracy reconstruction of lower sub grade parameters by using $\left|\dot{S}_{e}\left(\omega_{i}, \vec{P}\right)\right|$.
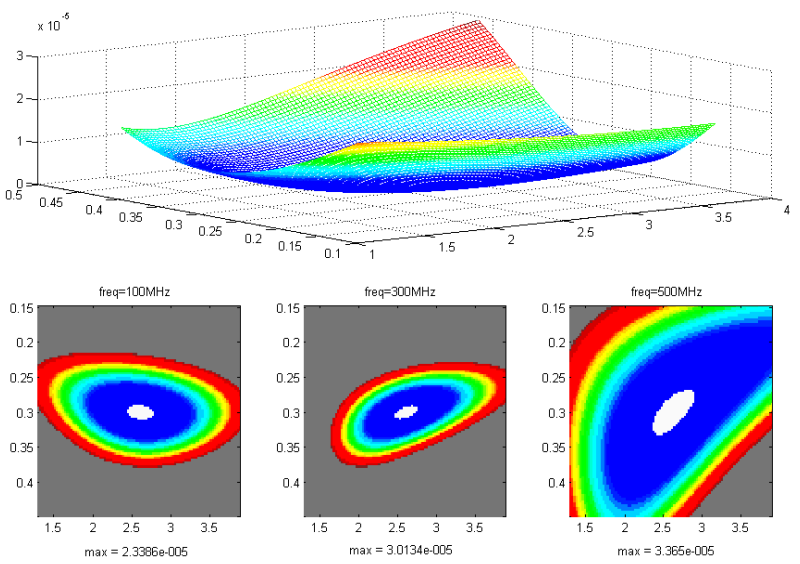

Figure 8: Influence of dielectric permittivity $\varepsilon_{1}^{\prime}$ and thickness $\mathrm{h}_{1}$ of the first layer on the aim function by using $\dot{S}_{e}\left(\omega_{i}, \vec{P}\right)$. 
In the upper part of each figure there is a three-dimensional view of the dependence of the aim function on two parameters calculated for $f_{\max }=300$ $\mathrm{MHz}$, and levels of aim functions are shown in the lower part of the figures for three values of maximal frequency $f_{\max }=100,300$ and $500 \mathrm{MHz}$. The threshold value $\alpha$ was used for the minimal level. In all these diagrams the value $\alpha$ was used for aim functions, and was calculated using $\mathrm{K}=2000$.
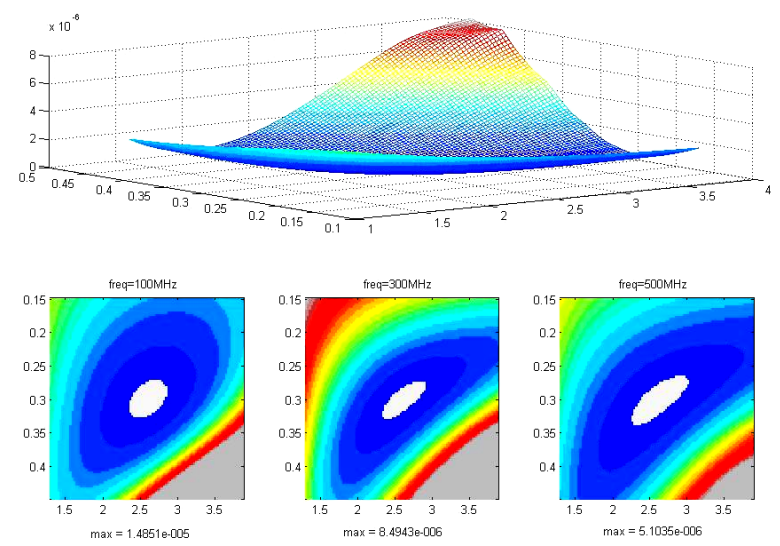

Figure 9: Influence of dielectric permittivity $\varepsilon_{1}^{\prime}$ and thickness $h_{1}$ of the first layer on the aim function by using $\left|\dot{S}_{e}\left(\omega_{i}, \vec{P}\right)\right|$.

The geometric forms of the white areas are ellipses (fig. 8 and fig. 9), but of different sizes. Comparative analysis of these areas allows us to make the following conclusion:

a. dielectric permittivity and thickness of layers heavily affect the values of aim functions;

b. calculation of the aim functions for $f_{\max }>300 \mathrm{MHz}$ increases the size and compression of the ellipses, which means that in order to calculate aim functions it is crucial, that $f_{\text {max }}$ is less than $300 \mathrm{MHz}$;

c. aim function with a complex spectral density is more informative, because areas taking values less than $\alpha$ are smaller in size, i.e. corresponding ranges of $h_{1}$ and $\varepsilon_{1}^{\prime}$ on the diagrams for using $\dot{S}_{e}\left(\omega_{i}, \vec{P}\right)$ are less than those for using $\left|\dot{S}_{e}\left(\omega_{i}, \vec{P}\right)\right|$;

d. values of aim function less than $\alpha$, are obtainable under multidirectional changes of dielectric permittivity and thickness of the layers against the modelled values of the parameters of the layers, which can lead to errors in parameters reconstruction of the probed medium;

e. influence of the electrical conductivity of the layers on the values of the aim functions is negligible. 
Optimal values of parameters and conditions of genetic algorithm use for searching of global minimum of aim function $\Phi$ are as follows:

- binary coding alphabet of an estimated parameter;

- number of bit per parameter is 8 ;

- $\quad$ probability of crossing over is 0,9 ;

- $\quad$ probability of mutation is 0,05 ;

- method of selection is roulette;

- admitted number of population into generation with the aim of functional means improvement is 500 .

The coefficient $K$ should be equal to several thousands to reduce the relative error of parameter reconstruction and the time of solving the inverse problem. However, the increase of coefficient $K$ increases the duration of the inverse problem solution.

\section{Conclusions}

The main results are as follows:

- the relative errors of parameter reconstruction do not exceed a few percent using complex values of the reflected signal spectral constituents;

- optimal parameters and conditions of genetic algorithm application for solving the inverse problem have been obtained;

- results of the work can be used for detection and identification of inner zones and objects of roadway coverage.

\section{Acknowledgements}

The paper is written with the financial assistance of European Social Fund. Project Nr. 2009/0159/1DP/1.1.2.1.2/09/IPIA/VIAA/006 (The Support in Realisation of the Doctoral Programme "Telematics and Logistics" of the Transport and Telecommunication Institute)

\section{References}

[1] Haas R., Pavement Design and Management Guide, Transportation Association of Canada, Ottawa, Ontario, 125-131, 1997.

[2] Rexford M. Morey., Ground Penetrating Radar for Evaluating Subsurface Conditions for Transportation Facilities (Synthesis of Highway Practice). National Academy Press, Washington D.C, 1998.

[3] Ground penetrating radar: theory and applications. Editor Harry M. Jol. Elsevier Science, 2009.

[4] Krainyukov A., Kutev V. Model-based results of inverse problem solution for radar monitoring of roadway coverage. Proc. of the Int. Conf. Modelling of Business, Industrial and Transport Systems, Riga, Latvia. Riga: Transport and Telecommunication Institute, pp.169-176, 2008.

[5] King R.W.P., Smith G.S. Antennas in Matter. Fundamentals, Theory, and Applications. The MIT Press, 1981. 